\title{
KCNMB3 in spinal microglia contributes to the generation and maintenance of neuropathic pain in mice
}

\author{
KAZUHISA IMARI $^{1,2}$, YUKA HARADA $^{2}$, JING ZHANG $^{2}$, YOSHIHIDE MORI $^{1}$ and YOSHINORI HAYASHI ${ }^{2,3}$ \\ ${ }^{1}$ Department of Oral and Maxillofacial Surgery, Graduate School of Dentistry; ${ }^{2}$ Department of Aging Science \\ and Pharmacology, Faculty of Dental Science, Kyushu University, Fukuoka 812-8582, Japan
}

Received March 29, 2019; Accepted July 1, 2019

DOI: $10.3892 /$ ijmm.2019.4279

\begin{abstract}
Neuropathic pain is one of most intense types of chronic pain. Numerous studies investigating neuropathic pain have described the critical involvement of microglia in the spinal cord. Previous studies have indicated that activation of large conductance $\mathrm{Ca}^{2+}$-activated $\mathrm{K}^{+}(\mathrm{BK})$ channels contributes to microglial activation in the spinal dorsal horn (SDH) and the generation of neuropathic pain. However, the specific role of BK channels in spinal microglia in neuropathic pain has not been fully addressed in previous studies, as BK channel inhibitors were used to inhibit microglial BK channel based on their inhibitory kinetics. We previously identified that $\mathrm{Ca}^{2+}$-activated $\mathrm{K}^{+}$channel $\beta 3$ auxiliary subunit (KCNMB3), which is an auxiliary subunit of BK channels and regulates gating properties of the channel, is exclusively expressed in microglia in the spinal cord. The present study analyzed the role of BK channels in spinal microglia in neuropathic pain using a spinal microglia-specific BK channel knockdown method, with intrathecal injection of KCNMB3 small interfering RNA. Neuropathic pain was significantly attenuated in KCNMB3 knockdown mice. Increases in the number of microglia in the SDH following nerve injury were attenuated by KCNMB3 knockdown. Furthermore, increased levels of pain-associated
\end{abstract}

Correspondence to: Dr Yoshinori Hayashi, Department of Aging Science and Pharmacology, Faculty of Dental Science, Kyushu University, 3-1-1 Maidashi, Higashi-ku, Fukuoka 812-8582, Japan

E-mail: yoshinor@dent.kyushu-u.ac.jp

${ }^{3}$ Present address: Department of Physiology, Nihon University School of Dentistry, Tokyo 101-8310, Japan

E-mail: hayashi.yoshinori@nihon-u.ac.jp

Abbreviations: BDNF, brain-derived neurotrophic factor; $\mathrm{BK}$, large conductance $\mathrm{Ca}^{2+}$-activated $\mathrm{K}^{+}$channels; IL, interleukin; IRF, interferon regulatory factor; LPA, lysophosphatidic acid; mRNA, messenger RNA; PWT, paw withdrawal threshold; SDH, spinal dorsal horn; siRNA, small interfering RNA; TLR, toll-like receptor; TNF, tumor necrosis factor

Key words: neuropathic pain, microglia, large conductance $\mathrm{Ca}^{2+}$-activated $\mathrm{K}^{+}$channel, $\mathrm{Ca}^{2+}$-activated $\mathrm{K}^{+}$channel $\beta 3$ auxiliary subunit, small interfering RNA molecules in the SDH were attenuated in KCNMB3 knockdown mice. Attempts were also made to analyze the effects of KCNMB3 knockdown on chronic pain. KCNMB3 knockdown ameliorated chronic pain and inhibited the expression levels of pain-associated molecules in the SDH. The results from the present study suggested that BK channels modulated the activation state of spinal microglia, and that KCNMB3 is a potential therapeutic target for neuropathic pain.

\section{Introduction}

Neuropathic pain is a chronic pain condition that may develop following damage, injury or dysfunction of nerves due to trauma, surgery, disease or chemotherapy. The pain has been described as a burning, shooting or crawling sensation, or similar to electric shocks. Slight touch may induce severe pain in patients, thereby seriously impairing quality of life. However, currently prescribed therapeutics for neuropathic pain have been insufficient for pain relief, and the development of new analgesics is challenging. Accumulating evidence from animal models of neuropathic pain indicate that microglia in the spinal dorsal horn $(\mathrm{SDH})$ have a critical role in the generation of neuropathic pain following peripheral nerve injury (1-3). Microglia immediately transform into an activated phenotype following peripheral nerve injury and subsequently initiate neuropathic pain. Activated microglia induce several pain-associated factors including purinergic receptor P2X4 (P2RX4), brain-derived neurotrophic factor (BDNF), interferon regulatory factor 8 (IRF8), and pro-inflammatory cytokines $(1,2,4,5)$, which sustain higher levels of neuronal activity in the SDH.

Activation of microglia manifests as hypertrophy of the cells' processes, and increased cell density and production of cytokines. Profound activation of microglia is frequently observed in the SDH after peripheral nerve injury (1-3). We previously identified that ketamine, a classical painkiller, inhibited not only N-methyl-D-aspartic acid receptors in neurons, a canonical target of ketamine, but also large conductance $\mathrm{Ca}^{2+}$-activated $\mathrm{K}^{+}(\mathrm{BK})$ channels in microglia (3). Blockade of $\mathrm{BK}$ channel function in microglia by selective inhibitors may attenuate the generation of neuropathic pain (3). However, a previous investigation did not rigorously address the specific role of microglial BK channels in vivo as BK channel blockers, not microglia-selective gene manipulation, 
were used to inhibit BK channels in microglia based on their inhibitory kinetics (3). BK channels are ubiquitously expressed in several tissues (6). Therefore, specific inhibition of microglial BK channels is required for understanding the mechanisms of neuropathic pain. The activation of microglia and subsequent generation of pain is not restricted in neuropathic pain models. Long-term opioid treatment in mice alters microglial phenotype, which induces pain hypersensitivity (7). Neuropathic pain and opioid-induced hyperalgesia share similar mechanisms that are mediated by microglial activation including membrane translocation of P2RX4 from lysosomes and subsequent induction of BDNF (7). We previously demonstrated that BK channels in spinal microglia are also activated following long-term morphine treatment (8). In particular, the $\mathrm{Ca}^{2+}$-activated $\mathrm{K}^{+}$channel $\beta 3$ auxiliary subunit (KCNMB3), one of a family of 4 auxiliary $\beta(\beta 1-\beta 4)$ subunits, was exclusively expressed in microglia in the spinal cord according to single-cell polymerase chain reaction (PCR) analysis (8). Furthermore, morphine-induced hyperalgesia was markedly attenuated in spinal cord specific-KCNMB3 knockdown mice (8). Therefore, the KCNMB3 knockdown method enables the investigation of the specific role of BK channels in spinal microglia during neuropathic pain.

BK channels contribute to an 'emergency brake' that regulates excessive generation of action potentials and transmitter release, and are broadly distributed throughout the body (6). BK channels are formed by a pore-forming $\alpha$ subunit and 4 types of auxiliary $\beta$ subunits, which comprise a heterotetramer. The function of the BK channel is distinct among tissues due to tissue-specific expression patterns of $\beta$ subunits (9). The $\beta 1$ subunit is distributed in smooth muscle cells, whereas the $\beta 2-\beta 4$ subunits are expressed in the central nervous system $(9,10)$. In the spinal cord, we identified that KCNMB3 was exclusively expressed in microglia, and that knockdown of KCNMB3 abrogated the function of BK channels in microglia (8). Conversely, $\beta 2$ and $\beta 4$ subunits expressed in astrocytes and neurons, respectively, were not detected in spinal microglia (8). Therefore, it is possible to evaluate the role of BK channels in spinal microglia in vivo by manipulating the $K C N M B 3$ gene. In the present study, the role of BK channels in microglia during neuropathic pain were analyzed by in vivo knockdown of KCNMB3, a microglia-specific subtype of BK channels in the spinal cord.

\section{Materials and methods}

Animals. C57BL/6 mice (male, 8-10 weeks old; $\mathrm{n}=60$ ) were purchased from CLEA Japan, Inc. The mice were maintained in a $12 \mathrm{~h}$ light:dark cycle (light beginning at $08: 00$ ) at $22-25^{\circ} \mathrm{C}$ ambient temperature with food and water provided ad libitum. All mice were handled daily for 5 days prior to the initiation of the experiment to minimize their stress reactions to manipulation.

Surgical procedure and small interference RNA (siRNA) injection. The mice were anesthetized with isoflurane (2\%; Pfizer, Inc.) in $\mathrm{O}_{2}$ (11). The back skin was additionally anesthetized with $0.5 \%$ xylocaine $(5 \mathrm{mg} / \mathrm{kg})$, following removal of the back hair by shaving. If no response was observed against a noxious stimulus, including toe or tail pinch, the surgical procedure was initiated. The lumber 5 (L5) transverse process was identified and carefully removed with bone rongeurs. The L4 ventral ramus was carefully isolated and freed from the adjacent nerve, and then the L4 nerve was transected according to a previous method (3). The incision was washed with saline and closed. For the intrathecal injection of siRNA, KCNMB3 siRNA (\#1: MSS224455; \#2: MSS224454; \#3: MSS284619) or control siRNA (GC duplex negative control) were used. Each siRNA (20 pmol/5 $\mu \mathrm{l})$ was suspended in Lipofectamine RNAiMAX, and then injected intrathecally for 4 consecutive days according to a previously described method $(3,8)$. All reagents were purchased from Thermo Fisher Scientific, Inc. The knockdown efficacy of siRNA was examined by western blot analyses. In certain experiments, the intrathecal injection of siRNA was initiated at 5 days after nerve injury.

Western blot analysis. The L4 spinal dorsal horn was collected from mice that were treated with siRNAs. The specimens were lysed in lysis buffer [10 mM Tris- $\mathrm{HCl}$ (pH 7.4), $150 \mathrm{mM} \mathrm{NaCl}$, $1 \%$ Triton X-100, $0.5 \%$ NP-40, phosphatase and protease inhibitor cocktail] and mixed with Laemmli sample buffer. Protein concentration was determined using the Pierce Bicinchoninic Acid Protein Assay kit (Thermo Fisher Scientific, Inc.). Proteins $(30 \mu \mathrm{g})$ were loaded into each lane, separated by $12 \%$ SDS-PAGE and transferred to a PVDF membrane. The blots were blocked with $0.2 \%$ Tween-20 in TBS (TBS-T) containing 5\% Blocking One (Nacalai Tesque, Inc.) for $1 \mathrm{~h}$ at room temperature, and were then incubated with primary antibodies, diluted in TBS-T containing 5\% Blocking One, at $4^{\circ} \mathrm{C}$ overnight. The primary antibodies used were as follows: Anti-KCNMB3 mouse monoclonal antibody (1:2,000; cat. no. NBP2-12916; Novus Biologicals, LLC) and anti- $\beta$-actin mouse monoclonal antibody $(1: 5,000$; cat. no. ab8226; Abcam). After being washed with TBS-T, the membranes were incubated with horseradish peroxidase (HRP)-conjugated secondary antibody (1:1,000; cat. no. NA931; GE Healthcare) for $1 \mathrm{~h}$ at room temperature. The membrane-bound HRP-labeled antibodies were detected using Immobilon ECL Ultra Western HRP Substrate (Merck KGaA) and an image analyzer (LAS-4000; Fujifilm). The bands that were evaluated by apparent molecular size and were semi-quantified using Image J $1.51 \mathrm{j}$ software (National Institutes of Health). The band intensity was normalized to $\beta$-actin.

Behavioral testing. The mice were examined for mechanical hypersensitivity of the hind paw following L4 nerve transection. All mice were habituated to the testing environment for 3 days and were examined for mechanical allodynia. The room temperature remained stable at $22 \pm 1^{\circ} \mathrm{C}$. The mice were placed in an acrylic cylinder (6-cm diameter) with wire mesh floors and allowed to habituate to the environment for $1 \mathrm{~h}$. Calibrated von Frey filaments (0.02-2.0 g; North Coast Medical, Inc.) were applied to the midplantar surface of the hind paw $(3,8)$. The 50\% paw withdrawal thresholds were calculated using the up-down method (12). Behavioral tests were performed for 3 or 10 days. Following behavioral analyses, mice were euthanized with sodium pentobarbital $[200 \mathrm{mg} / \mathrm{kg}$, intraperitoneal (i.p.) injection]. Animal death was confirmed by cessation of respiration and heartbeat. 
Patch clamp. For this protocol, six mice were euthanized with sodium pentobarbital $(200 \mathrm{mg} / \mathrm{kg}$, i.p.) at 3 days after nerve injury. Animal death was confirmed by cessation of respiration and heartbeat. A block of the spinal cord from L3 to L5 was embedded in $1 \%$ agar. Transverse slices (200- $\mu \mathrm{m}$ thick) were cut from L4 segment with a vibratome (VT1000 S; Leica Biosystems Nussloch GmbH). Ice-cold artificial cerebrospinal fluid (ACSF) saturated with $95 \% \mathrm{O}_{2}$ and $5 \% \mathrm{CO}_{2}$ was used in slice preparation. ACSF consisted of $124 \mathrm{mM} \mathrm{NaCl}, 2.5 \mathrm{mM} \mathrm{KCl}, 1.24 \mathrm{mM} \mathrm{KH}_{2} \mathrm{PO}_{4}, 1.3 \mathrm{mM}$ $\mathrm{MgSO}_{4}, 2.4 \mathrm{mM} \mathrm{CaCl}_{2}, 10 \mathrm{mM}$ glucose, and $26 \mathrm{mM} \mathrm{NaHCO}_{3}$ (all from Sigma-Aldrich; Merck KGaA). Spinal slices were stained with $25 \mu \mathrm{g} / \mathrm{ml}$ Alexa Fluor 488 conjugated isolectin

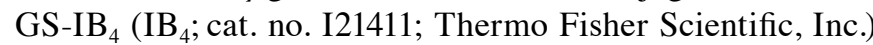
for $30 \mathrm{~min}$ at room temperature in ACSF. A whole-cell patch clamp recording was made from $\mathrm{IB}_{4}$-positive cells with marked branched processes located at lamina II-III in L4 spinal cord in the slice preparation. IB $_{4}$-positive cells were visually identified by the laser with a wavelength of $488 \mathrm{~nm}$ using an upright microscope equipped with a $\mathrm{x} 40$ water-immersion objective (Zeiss $\mathrm{GmbH})$. The external solution was ACSF. Patch electrodes were fabricated using a Sutter P-97 (Sutter Instrument) from borosilicate glass (1.5 mm outer diameter, $0.9 \mathrm{~mm}$ inner diameter; G-1.5; Narishige Scientific Instrument Laboratories). Patch pipettes were filled with internal solution containing $1 \%$ Lucifer Yellow $\mathrm{CH}$ dilithium salt (Sigma-Aldrich; Merck KGaA). Internal solution consisted of $120 \mathrm{mM} \mathrm{KCl}, 2 \mathrm{mM} \mathrm{MgCl}$, $10 \mathrm{mM}$ HEPES, $0.1 \mathrm{mM}$ BAPTA adjusted to $\mathrm{pH} 7.3$ with $\mathrm{KOH}$ (all from Sigma-Aldrich; Merck KGaA). Voltage ramps from -120 to $+30 \mathrm{mV}$ were applied for $300 \mathrm{msec}$ to induce BK currents. The data were acquired using Axopatch 200B amplifier, Digidata 1320 interface, and pClamp 9.0 software and analyzed with Clampfit 9 software (all from Molecular Devices, LLC) according to the previous methods $(3,8)$.

Immunohistochemistry. The mice were euthanized with sodium pentobarbital (200 mg/kg, i.p.). Animal death was confirmed by cessation of respiration and heartbeat. The mice were perfused transcardially with $0.1 \mathrm{M}$ phosphate buffer (PB), $\mathrm{pH} 7.4$, followed by $4 \%$ paraformaldehyde in $0.1 \mathrm{M}$ $\mathrm{PB}, 3$ and 10 days after nerve injury. The L4 spinal segments were fixed with $4 \%$ paraformaldehyde (PFA; Sigma-Aldrich; Merck KGaA) overnight at $4^{\circ} \mathrm{C}$. The L4 segments were further incubated with $30 \%$ sucrose (Sigma-Aldrich; Merck KGaA) overnight to protect cryolesions. The spinal cord slices $(14-\mu \mathrm{m}$ thick) were prepared by a CM1860 cryomicrotome (Leica Microsystems, Inc.). In some experiments, the slices were fixed with 4\% PFA following the patch clamp recording, after which, staining was performed according to the following method. Blocking was performed by $1 \%$ normal donkey serum (Jackson ImmunoResearch Laboratories, Inc.), 1\% BSA (Sigma-Aldrich; Merck KGaA) and $0.1 \%$ Triton X-100 (Sigma-Aldrich; Merck KGaA) in PBS for $1 \mathrm{~h}$. The slices were incubated with anti-Lucifer Yellow rabbit polyclonal antibody (1:50,000; cat. no. A-5750; Thermo Fisher Scientific, Inc.) or anti-ionized calcium-binding adapter molecule 1 (Iba1), a marker for microglia, rabbit polyclonal antibody (1:2,000; cat. no. 019-19741; FUJIFILM Wako Pure Chemical Corporation) for 7 days at $4^{\circ} \mathrm{C}$ or overnight at $4^{\circ} \mathrm{C}$, respectively. Following washing with PBS, the slices were incubated with donkey anti-rabbit IgG conjugated with Cy3 (cat. no. 711-165-152, 1:400; Jackson ImmunoResearch Laboratories, Inc.) or Alexa488 (711-545-152, 1:400; Jackson ImmunoResearch Laboratories, Inc.) for $2 \mathrm{~h}$ at $4^{\circ} \mathrm{C}$, and then mounted in Vectashield (Vector Laboratories, Inc.). The images were acquired on a Nikon $\mathrm{C} 2$ scanning confocal microscope using a x20 objective (NA 0.75; Nikon Corporation). Images were processed and analyzed using Image J $1.51 \mathrm{j}$ software (National Institutes of Health). The number of microglia in the SDH was counted. To analyze the microglial morphology, Sholl analysis was performed manually according to a previously described method (13): Concentric circles with $5 \mu \mathrm{m}$ increases in diameter were drawn around the soma and the number of processes crossing each circles was counted. All measurements were performed by an operator who was blinded to the identity of the sections.

Reverse transcription quantitative PCR (RT-qPCR) analyses. The total RNA was extracted from SDH samples with TRIsure (Bioline; BiCat $\mathrm{GmbH}$ ) according to the protocol of the manufacturer. RT was performed using a QuantiTect Reverse Transcription kit (Qiagen $\mathrm{GmbH}$ ) with $500 \mathrm{ng}$ extracted RNA, according to the manufacturer's protocol (DNA elimination: $42^{\circ} \mathrm{C}$ for $2 \mathrm{~min}$; RT: $42^{\circ} \mathrm{C}$ for $15 \mathrm{~min}$; inactivation of QuantiTect Reverse Transcriptase: $95^{\circ} \mathrm{C}$ for $3 \mathrm{~min}$ ). RT-qPCR was performed using a two-step protocol (initial denaturation: $95^{\circ} \mathrm{C}$ for $2 \mathrm{~min}$ and 45 cycles of denaturation: $95^{\circ} \mathrm{C}$ for $10 \mathrm{sec}$ and annealing: $60^{\circ} \mathrm{C}$ for $10 \mathrm{sec}$ ) and processed in triplicate with a Corbett Rotor-Gene RG-3000A Real-Time PCR System (Qiagen GmbH) using Thunderbird SYBR qPCR Mix $(10 \mu \mathrm{l})$ and 50X ROX reference dye $(0.4 \mu \mathrm{l}$; both from Toyobo Life Science) and cDNA template $(0.2 \mu \mathrm{l})$. The primers $(600 \mathrm{nM})$ for each gene are listed below. The data were analyzed by an RG-3000A software program (version Rotor-Gene 6.1.93; Corbett Life Science; Qiagen GmbH.). All values were normalized to $\beta$-actin expression. The $2^{-\Delta \Delta \mathrm{Cq}}$ method was used to calculate relative mRNA expression levels (14). The primer sequences are described as follows: P2RX4 forward, 5'-ACA ACGTGTCTCCTGGCTACAAT-3'; P2RX4 reverse, 5'-GTC AAACTTGCCAGCCTTTCC-3'; BDNF forward, 5'-TACCTG GATGCCGCAAACAT-3'; BDNF reverse, 5'-AGTTGGCCT TTGGATACCGG-3'; IRF8 forward, 5'-GAGCTGCAGCAA TTCTACGC-3'; IRF8 reverse, 5'-AAGGGTCTCTGGTGT GAGGT-3'; tumor necrosis factor- $\alpha$ (TNF- $\alpha$ ) forward, 5'-GTG GAACTGGCAGAAGAGGC-3'; TNF- $\alpha$ reverse, 5'-AGACAG AAGAGCGTGGTGGC-3'; interleukin-1 $\beta$ (IL-1 $\beta$ ) forward, 5'-CTGTGTCTTTCCCGTGGACC-3'; IL-1 $\beta$ reverse, 5'-CAG CTCATATGGGTCCGACA-3'; $\beta$-actin forward; 5'-AGAGGG AAATCGTGCGTGAC-3'; and $\beta$-actin reverse, 5'-CAATAG TGATGACCTGGCCGT-3'.

Statistical analyses. The data are represented as the mean \pm standard error of the mean. Statistical analyses of the results were performed with Student's unpaired t-test and one-way analysis of variance (ANOVA) with post-hoc Dunnett's or Tukey's test, and two-way ANOVA with post-hoc Tukey's test using the GraphPad Prism7 (GraphPad Software, Inc.) software package. $\mathrm{P}<0.05$ was considered to indicate a statistically significant difference. 
A

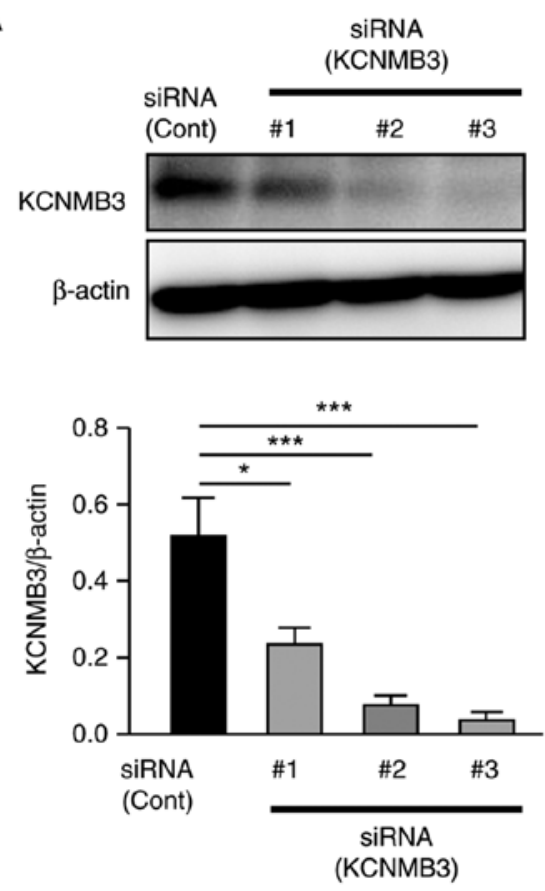

B
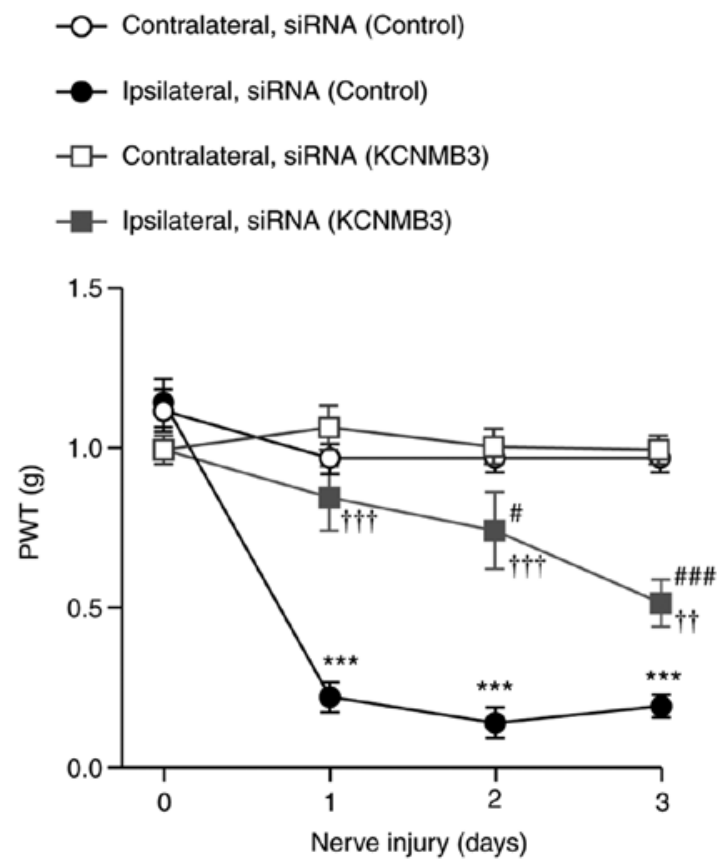

Figure 1. KCNMB3 knockdown attenuates the generation of neuropathic pain. (A) Knockdown efficacy of KCNMB3 siRNA in the spinal dorsal horn. Western blot analysis for KCNMB3 in the L4 spinal dorsal horn. The bar chart represents densitometric analysis of KCNMB3 protein levels normalized with $\beta$-actin. $\mathrm{n}=3$ mice/group. Data were analyzed by one-way ANOVA with Dunnett's post-hoc test, ${ }^{*} \mathrm{P}<0.05$ and ${ }^{* * *} \mathrm{P}<0.001$ vs. siRNA (Cont). (B) Time course of PWT following nerve injury. KCNMB3 siRNA was injected intrathecally into naïve mice prior to nerve injury. $\mathrm{n}=4$ mice/group. Data were analyzed by two-way ANOVA followed by Tukey's post-hoc test. ${ }^{* * *} \mathrm{P}<0.001$ vs. Contralateral, siRNA (Control); ${ }^{\dagger} \mathrm{P}<0.01$ and ${ }^{\dagger \dagger} \mathrm{P}<0.001 \mathrm{vs}$. Ipsilateral, siRNA (Cont); ${ }^{\#} \mathrm{P}<0.05$ and ${ }^{\# \#} \mathrm{P}<0.01$ vs. Contralateral, siRNA (KCNMB3). Data are presented as the mean \pm standard error of the mean. KCNMB3, $\mathrm{Ca}^{2+}$-activated $\mathrm{K}^{+}$channel $\beta 3$ auxiliary subunit; ANOVA, analysis of variance; siRNA, small interfering RNA; PWT, paw withdrawal threshold; Cont, control.

\section{Results}

In vivo knockdown of KCNMB3 attenuates the generation of neuropathic pain. To evaluate the role of BK channels in spinal microglia on neuropathic pain, in vivo knockdown experiments were performed by intrathecal injection of siRNA, as microglia in the SDH, as well as several brain regions, are activated after peripheral nerve injury (15). In a previous study, we identified that the expression pattern of KCNMB3 was restricted in microglia in the spinal cord (8). Therefore, KCNMB3 siRNA was used to silence microglial BK channels in the spinal cord. KCNMB3 siRNA ( 20 pmol, $5 \mu \mathrm{l})$ was injected into the intrathecal space of naïve mice for 4 consecutive days; the L4 segment of the SDH was then collected. The knockdown efficacy of KCNMB3 siRNAs was examined using western blot analyses. Among the siRNAs for KCNMB3, \#3 siRNA (MSS284619) resulted in almost complete depletion of KCNMB3 protein expression in the SDH (Fig. 1A). Spinal microglia-specific knockdown of KCNMB3 was successful; therefore, the \#3 siRNA was used in subsequent experiments. Nerve-injured mice exhibited a significant decreased in paw withdrawal threshold (PWT) to mechanical stimulation applied to the hind paw following nerve injury (Fig. 1B). Conversely, the decrease in PWT following nerve injury was significantly attenuated in the KCNMB3 knockdown mice compared with the control siRNA-treated group (Fig. 1B). To additionally evaluate the function of BK channels in spinal microglia during neuropathic pain, whole-cell patch clamp analyses from spinal microglia were performed 3 days after nerve injury. Spinal cord slices from nerve-injured mice were stained with $\mathrm{IB}_{4}$ to visualize the microglia. Although patch pipette was applied to $\mathrm{IB}_{4}{ }^{+}$cells, which have branched processes (Fig. $2 \mathrm{~A}$ ), $\mathrm{IB}_{4}$ preferentially labels nonpeptidergic primary afferent fibers that terminate in laminas II of the SDH (16). Lucifer yellow was introduced through the recording pipette to verify that the recorded cells were microglia and not primary afferent fibers. Immunohistochemical analyses using anti-Lucifer yellow antibodies revealed that the recorded cells exhibited the characteristic structure of microglia that have soma with branched processes, as demonstrated by the Z-stack image in Fig. 2A, indicating successful patch clamp recordings from microglia and not primary afferent fibers in the SDH. Ipsilateral spinal microglia at 3 days after nerve injury exhibited large outward currents at $+30 \mathrm{mV}$, in contrast to those in the sham-operated mice (Fig. 2B), similar to observations from previous studies (3). These current activations were significantly weakened in the spinal microglia from the nerve-injured KCNMB3 knockdown mice (Fig. 2B). The Sholl analysis of the microglial processes was performed to examine the morphological changes of the microglia in the SDH. Morphological changes associated with microglial activation, which were characterized by retraction of their processes, in nerve-injured mice were prevented by KCNMB3 knockdown (Fig. 2C).

KCNMB3 knockdown prevents microglial activation following nerve injury. The activation profile of microglia following nerve injury was subsequently analyzed. The L4 spinal cord was collected 3 days after nerve injury and stained with anti-Iba1 antibody, a microglia marker. The immunoreactivity of Iba1 in the ipsilateral side of SDH was significantly increased compared 
A IB4/Lucifer Yellow
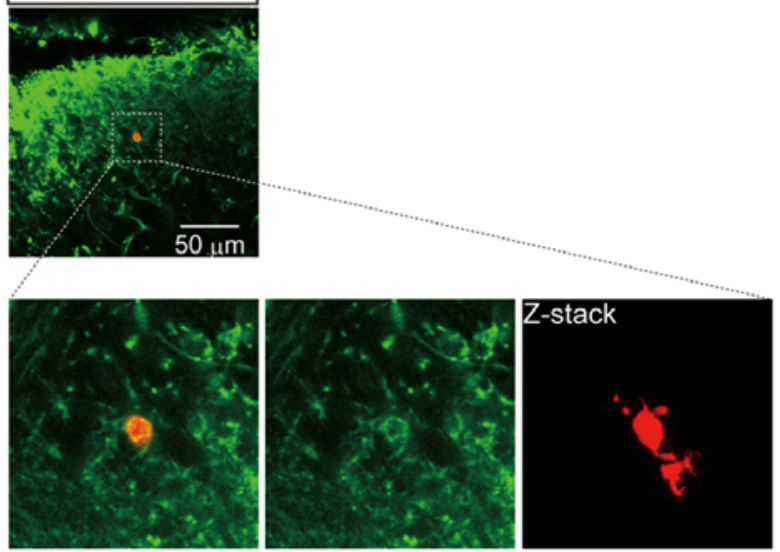

$\mathrm{B} \square$ Sham

Ipsi, siRNA (Cont)

Ipsi, siRNA (KCNMB3)

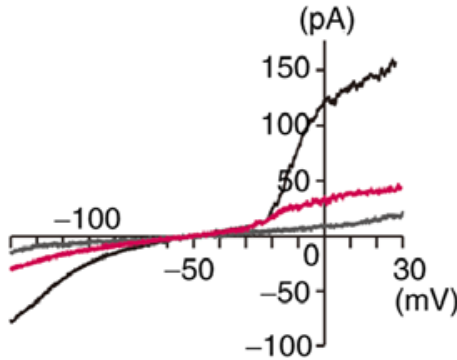

Ipsi

C
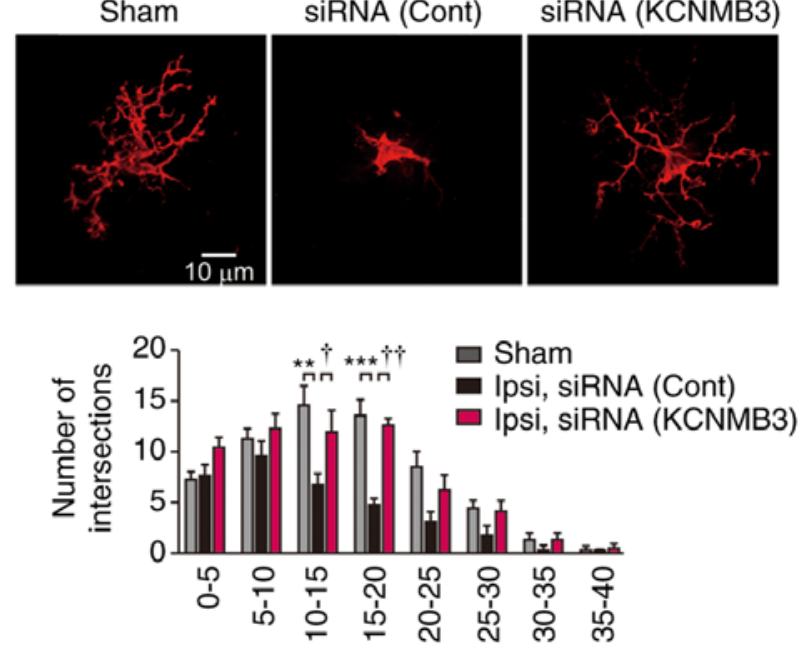

Distance from soma $(\mu \mathrm{m})$

Figure 2. Patch clamp recording from $\mathrm{IB}_{4}$-positive cells in the spinal dorsa horn. (A) Lucifer yellow was introduced into $\mathrm{IB}_{4}$-positive cells through patch pipette during recording, and then stained with anti-Lucifer yellow antibody. Upper panel demonstrates merged image of $\mathrm{IB}_{4}$ and anti-Lucifer yellow staining of the spinal cord slices from nerve-injured mice on day 3 . Lower panels demonstrate enlarged image of the inset (square). The image on the right-hand side in the lower panel demonstrates the Z-stack of Lucifer yellow-positive cells. Scale bar, $50 \mu \mathrm{m}$. (B) Representative BK currents in spinal microglia elicited by voltage ramps. The bar chart represents statistical analysis of BK currents at $+30 \mathrm{mV} . \mathrm{n}=6$ cells from 3 mice each. Data were analyzed by one-way ANOVA followed by Tukey's post-hoc test. ${ }^{* * *} \mathrm{P}<0.001$ ${ }^{\dagger+} \mathrm{P}<0.001$. (C) Representative Z-stack images of Lucifer yellow positive microglia of sham-, siRNA (cont)-treated nerve-injured mice, and siRNA (KCNMB3)-treated nerve-injured mice. Scale bar, $10 \mu \mathrm{m}$. Lower panel indicated Sholl analysis of microglia in the SDH. $n=6$ cells from 3 mice each. Data were analyzed by two-way ANOVA followed by Tukey's post-hoc test ${ }^{* *} \mathrm{P}<0.01$ and ${ }^{* * *} \mathrm{P}<0.001$. ${ }^{\dagger} \mathrm{P}<0.05$ and ${ }^{\dagger \dagger} \mathrm{P}<0.01$. Data are presented as the mean \pm standard error of the mean. IB4, isolectin GS-IB4; ANOVA, analysis of variance; Ipsi, ipsilateral; siRNA, small interfering RNA; Cont, control; $\mathrm{KCNMB} 3, \mathrm{Ca}^{2+}$-activated $\mathrm{K}^{+}$channel $\beta 3$ auxiliary subunit. with that of the contralateral side (Fig. 3A). Microglial density was also increased in the ipsilateral side of SDH compared with the contralateral side (Fig. 3B). Conversely, KCNMB3 knockdown markedly decreased Iba1 immunofluorescence and microglial density in the ipsilateral side of SDH compared with that of control siRNA-treated group (Fig. 3A and B). The effects of KCNMB3 siRNA on pain-associated molecules were additionally analyzed in the SDH. The messenger RNA (mRNA) levels on the ipsilateral side of the SDH were normalized with those on the contralateral side. Increased expression levels of mRNAs for P2X4R, BDNF, IRF8, IL-1 $\beta$ and TNF- $\alpha$ in the SDH following nerve injury were significantly attenuated by KCNMB3 knockdown (Fig. 4). These results indicated that KCNMB3 knockdown prevented activation of microglia.

Gene silencing of KCNMB3 in microglia ameliorates neuropathic pain. To investigate the role of KCNMB3 in chronic pain, intrathecal injection of KCNMB3 siRNA was initiated at day 5 after nerve injury. Mechanical allodynia was gradually but significantly alleviated by daily injection of KCNMB3 siRNA (Fig. 5A). The L4 spinal cord was collected 10 days after nerve injury. Iba1 immunoreactivity and microglia density in the SDH were significantly decreased by intrathecal injection of KCNMB3 siRNA (Fig. 5B and C). In addition, increased expression levels of mRNAs for pain-associated molecules, including P2X4R, BDNF, IRF8, IL-1 $\beta$ and TNF- $\alpha$, in the ipsilateral side of the $\mathrm{SDH}$ were also suppressed by KCNMB3 siRNA treatment (Fig. 6). These results suggest that KCNMB3 knockdown shifts microglia in a quiescent state.

\section{Discussion}

Spinal microglia are known to be involved in pain, including nerve injury-, cancer- and diabetic-induced neuropathic pain and opioid-induced hyperalgesia (17). Microglia-derived molecules, including IL- $1 \beta$ and TNF- $\alpha$, sustain the pain state by the facilitation of excitatory neurotransmission and decrease of inhibitory neurotransmission in lamina II neurons in the spinal cord (4). In addition, microglia-derived BDNF leads to excitatory action of $\gamma$-aminobutyric acid via a depolarizing shift in the anion reversal potential (2). Due to amelioration of allodynia by neutralizing IL-1 $\beta$ or quenching $\operatorname{BDNF}(2,4)$, the functional inhibition of reactive microglia has significance in pain relief. The major results from the present study revealed that microglia-specific inhibition of BK channels resulted in a decreased production of pain-associated molecules and attenuation of neuropathic pain.

The family of $\mathrm{Ca}^{2+}$-activated $\mathrm{K}^{+}$channels is comprised of small conductance, intermediate conductance, and large conductance (i.e. BK) channels. Among the 3 types of $\mathrm{Ca}^{2+}$-activated $\mathrm{K}^{+}$channels, BK channels are solely expressed in microglia (18). BK channels are involved in the regulation of physiological processes, including neuronal excitability and neurotransmitter release. However, the role of BK channels in microglia remains to be completely elucidated. Concordant with previous observations that the activation of $\mathrm{K}^{+}$channels are observed in reactive microglia $(19,20)$, BK channels in microglia are activated during neuropathic pain, which is involved in the production of IL- $1 \beta$ and $\operatorname{BDNF}(3,8)$. Previous studies have revealed that $\mathrm{BK}$ channel inhibitors impede the 
A
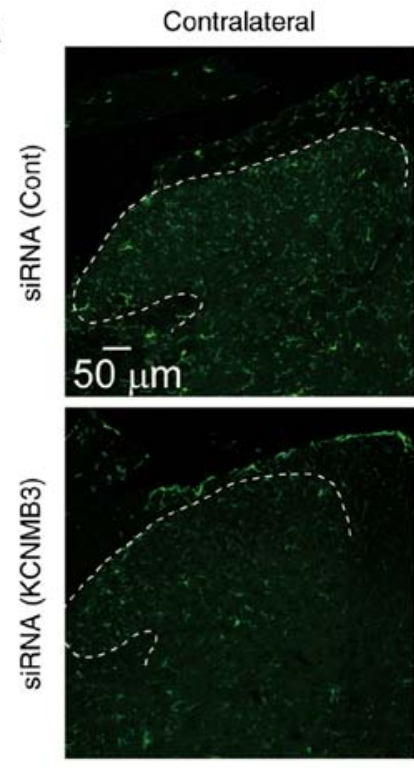
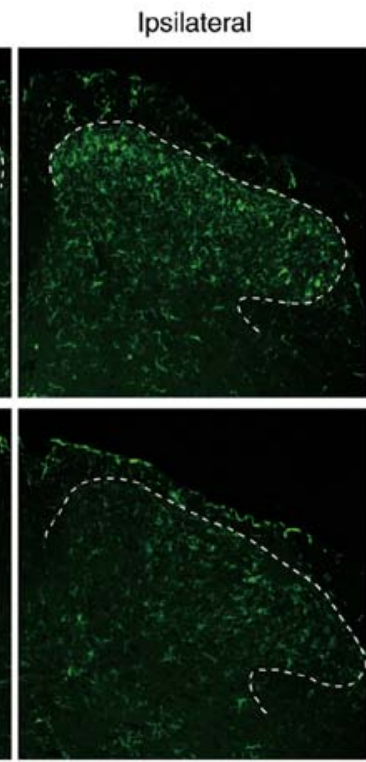

B

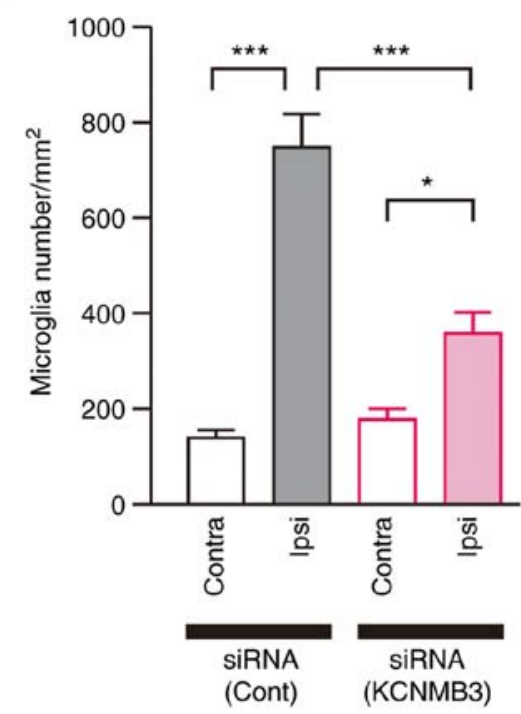

Figure 3. KCNMB3 knockdown attenuates the activation of spinal microglia following nerve injury. (A) Immunofluorescence of ionized calcium-binding adapter molecule 1, a marker of microglia, 3 days after nerve injury. KCNMB3 siRNA was injected intrathecally prior nerve injury. The dashed lines indicate the border of white and gray matter of the spinal dorsal horn. Scale bar, $50 \mu \mathrm{m}$. (B) The number of microglia in the spinal dorsal horn. Bars represent the microglia number. $\mathrm{n}=4$ mice/group. Data were analyzed by two-way analysis of variance followed by Tukey's post-hoc test. ${ }^{*} \mathrm{P}<0.05$ and ${ }^{* * *} \mathrm{P}<0.001$. Data are presented as the mean \pm standard error of the mean. KCNMB3, $\mathrm{Ca}^{2+}$-activated $\mathrm{K}^{+}$channel $\beta 3$ auxiliary subunit; siRNA, small interfering RNA; Contra, contralateral; Ipsi, ipsilateral; Cont, control.

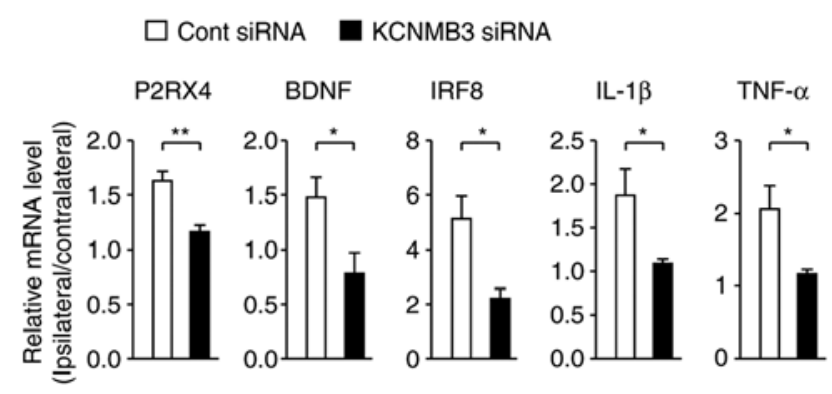

Figure 4. KCNMB3 knockdown prevents induction of neuropathic painassociated molecules. Relative expression levels of mRNAs for P2RX4, BDNF, IRF8, IL-1 $\beta$ and TNF- $\alpha$ in the SDH 3 days after nerve injury. The mRNA levels in the ipsilateral side of SDH were normalized to those in the contralateral side. Bars represent mRNA levels. $n=3-4$ mice/group, unpaired t-test. ${ }^{*} \mathrm{P}<0.05$ and ${ }^{* *} \mathrm{P}<0.01$. Data are presented as the mean \pm standard error of the mean. $\mathrm{KCNMB} 3, \mathrm{Ca}^{2+}$-activated $\mathrm{K}^{+}$channel $\beta 3$ auxiliary subunit; P2RX4, purinergic receptor P2X4, BDNF, brain-derived neurotrophic factor; IRF8, interferon regulatory factor 8 ; IL-1 $\beta$, interleukin-1 $\beta$; TNF- $\alpha$, tumor necrosis factor- $\alpha$; SDH, spinal dorsal horn; cont, control; siRNA, small interfering RNA.

secretion process of IL-1 $\beta$ from both lipopolysaccharide [Toll-like receptor (TLR) 4 agonist]- and Pam ${ }_{3}$ CSK4 (TLR2 agonist)-stimulated human monocytes (20). $\mathrm{K}^{+}$efflux via BK channels is involved in the activation of the NACHT, LRR and PYD domain-containing protein 3 inflammasome, which is independent of pannexin-1 or $\mathrm{P} 2 \mathrm{X}$ purinoceptor 7 receptor pathways (21). Similarly, activation of BK channels contributes to the inflammatory response in human macrophages (22). Therefore, BK channels contribute to the production of pro-inflammatory molecules in microglia. BK currents are observed in resting microglia of juvenile mice (20); however, their expression levels are decreased in the microglia of young adult mice (23). Regardless of silent of BK channels in resting microglia, an increase in intracellular $\mathrm{Ca}^{2+}$ concentration through patch pipette caused robust activation of $\mathrm{BK}$ channels (8). Microglia in the developmental stage are highly active, and contribute to synaptic pruning (24) and survival of cortical neurons (25). Considering the aforementioned data, activation of BK channels may contribute to sustaining the active state of microglia.

Single-cell PCR analysis demonstrated that only KCNMB3 and not KCNMB1, KCNMB2 or KCNMB4, is exclusively expressed in spinal microglia and primary cultured microglia (8). However, it cannot be concluded that the current activation of $\mathrm{BK}$ channels in spinal microglia following nerve injury is due to an increase in their numbers. Microgliosis in the SDH following nerve injury impedes the amount of KCNMB3 protein in a microglia. Furthermore, there is a requirement for the evaluation of KCNMB3 protein levels in a microglial cell isolated from the SDH via fluorescence-activated cell sorting in future studies. Single-channel recording demonstrated that opening of the BK channel coupled with KCNMB3 was observed at negative potentials (26). By contrast, KCNMB1, KCNMB2 and KCNMB4 are not activated at negative potentials $(9,27)$. In the present study, it was observed that spinal microglia following nerve injury exhibited large inward currents at negative potentials. Considering the gating properties of the KCNMB protein family, it is conceivable that functional KCNMB1, KCNMB2 and KCNMB4 are not expressed in spinal microglia following nerve injury.

Microgliosis in the SDH was observed at 2 months after nerve injury when pain was completely recovered (28). We hypothesized that KCNMB3 siRNA injection during the maintenance phase of neuropathic pain would not affect microgliosis in the SDH. Contrary to our hypothesis, the number of spinal microglia observed following nerve injury was decreased due to KCNMB3 siRNA treatment. The 


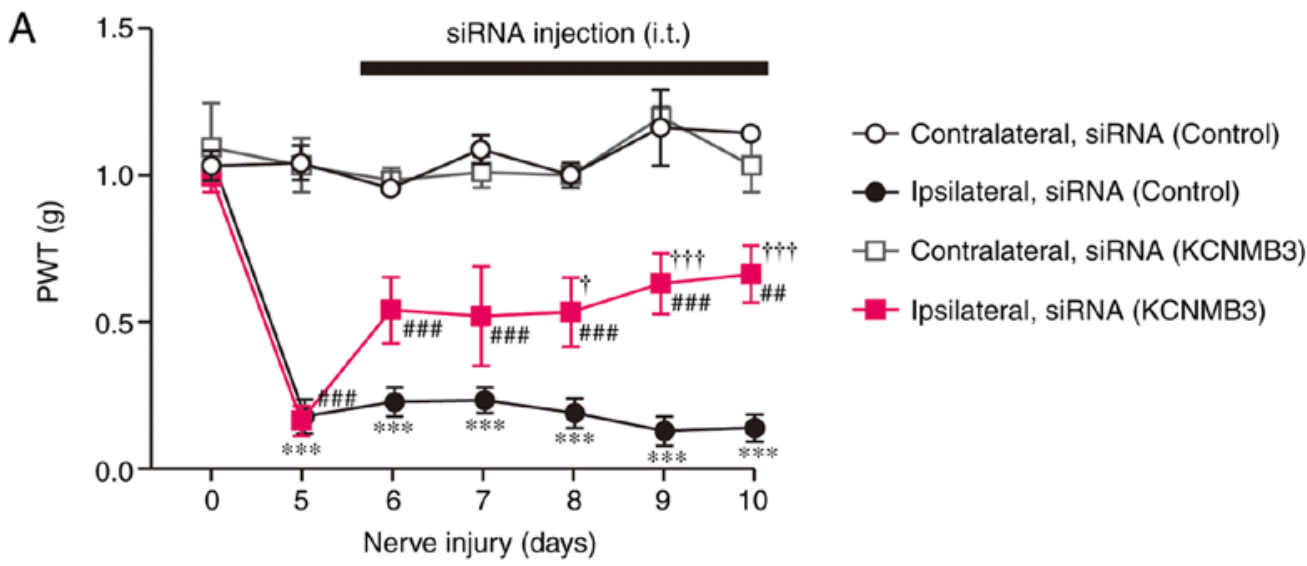

B
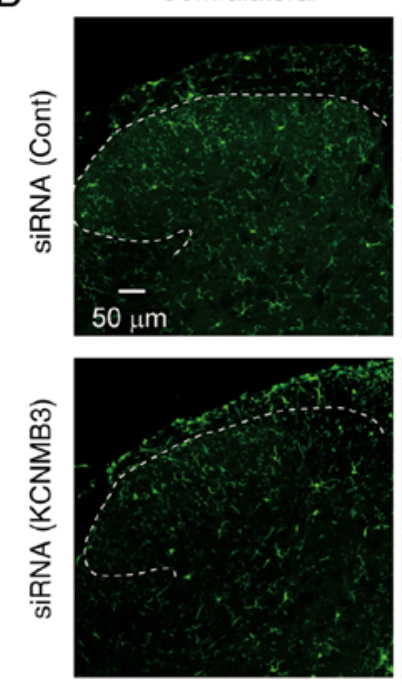

Ipsilateral
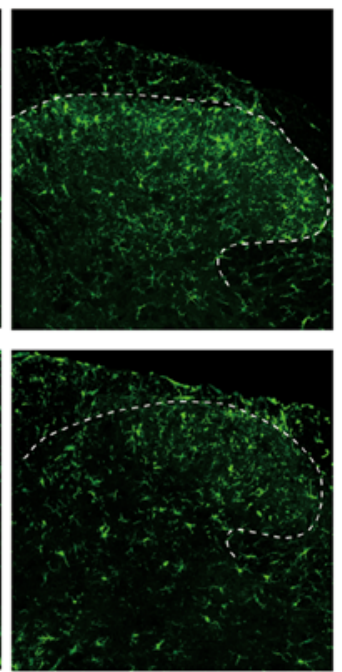

C

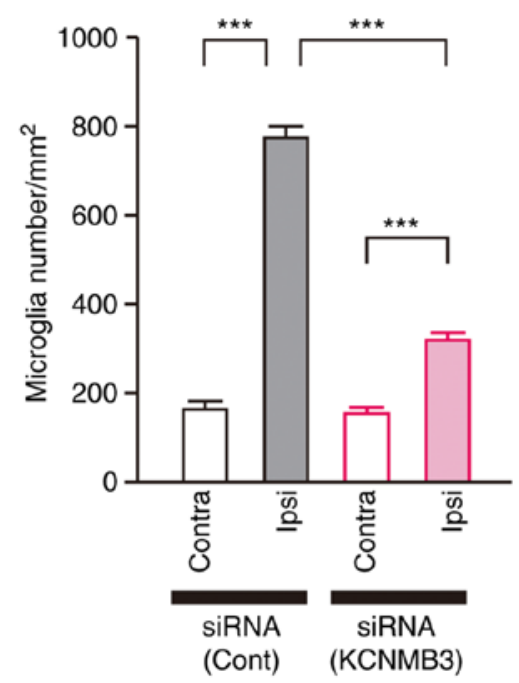

Figure 5. KCNMB3 knockdown ameliorates chronic pain. (A) Time course of PWT following nerve injury. Intrathecal injection of KCNMB3 siRNA was initiated at 5 days after nerve injury. $\mathrm{n}=4$ mice/group. Data were analyzed using two-way ANOVA followed by Tukey's post-hoc test. ${ }^{* * *} \mathrm{P}<0.001 \mathrm{vs}$. Contralateral, siRNA (Control). ${ }^{~} \mathrm{P}<0.05$ and ${ }^{\dagger \dagger t} \mathrm{P}<0.001$ vs. Ipsilateral, siRNA (Control). ${ }^{\# \#} \mathrm{P}<0.01$ and ${ }^{\# \# \#} \mathrm{P}<0.001$ vs. Contralateral, siRNA (KCNMB3). (B) Immunofluorescence of ionized calcium-binding adapter molecule 1, a marker of microglia, 10 days after nerve injury. KCNMB3 siRNA was injected intrathecally 5 days after nerve injury. Dashed lines indicate the border of white and gray matter of the spinal dorsal horn. Scale bar, $50 \mu \mathrm{m}$. (C) The number of microglia in the spinal dorsal horn. Bars represent microglia number. $\mathrm{n}=4$ mice/group. Data were analyzed by two-way ANOVA followed by Tukey's post-hoc test. ${ }^{* * *} \mathrm{P}<0.001$. Data are presented as the mean \pm standard error of the mean. $\mathrm{KCNMB} 3, \mathrm{Ca}^{2+}$-activated $\mathrm{K}^{+}$channel $\beta 3$ auxiliary subunit; PWT, paw withdrawal threshold; siRNA, small interfering RNA; cont, control; ANOVA, analysis of variance.

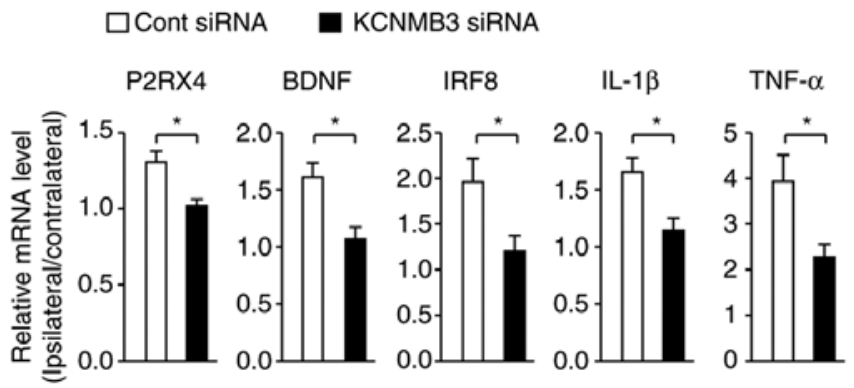

Figure 6. KCNMB3 knockdown inhibited the expression levels of neuropathic pain-associated molecules. Relative expression levels of mRNAs for P2RX4, BDNF, IRF8, IL-1 $\beta$ and TNF- $\alpha$ in the SDH 10 days after nerve injury. The mRNA levels in the ipsilateral side of the SDH were normalized with those in the contralateral side. Bars represent mRNA levels. $n=4$ mice/group. Data were analyzed using an unpaired Student's t-test. ${ }^{*} \mathrm{P}<0.05$. Data are presented as the mean \pm standard error of the mean. $\mathrm{KCNMB} 3, \mathrm{Ca}^{2+}$-activated $\mathrm{K}^{+}$ channel $\beta 3$ auxiliary subunit; P2RX4, purinergic receptor P2X4, BDNF, brain-derived neurotrophic factor; IRF8, interferon regulatory factor 8 ; IL-1 $\beta$, interleukin- $1 \beta$; TNF- $\alpha$, tumor necrosis factor- $\alpha$; SDH, spinal dorsal horn; cont, control; siRNA, small interfering RNA. mechanisms responsible for the decrease in the number of spinal microglia via KCNMB3 knockdown remain unknown. Microgliosis in the SDH following nerve injury has previously been hypothesized to occur as a result of microglial proliferation. BDNF is known to regulate the proliferation and survival of cultured microglia (29). Therefore, BDNF decrease following KCNMB siRNA injection may potentially affect the total number of microglia. However, microglial proliferation in the SDH peaks 3 days after nerve injury (13). Microglial proliferation during the induction phase of neuropathic pain may be attenuated by KCNMB3 siRNA injection. Alternatively, it is unlikely that KCNMB3 siRNA suppresses microglial proliferation during the maintenance phase of neuropathic pain. An additional mechanism for a decrease in the microglial density via KCNMB3 siRNA may be the reestablishment of the microglial network. Tay et al (30) demonstrated that microglial egress and apoptosis were observed during the resolution of microglial clusters. KCNMB3 siRNA may facilitate these processes and shift the 
stable microglial network. Its role in the microglial network should be assessed in future studies.

Changes in spinal density are associated with synaptic connectivity and behavior. Microglia-derived BDNF is one of the representative modulators of synaptic structure through the formation of glutamatergic synapses (31). Liu et al (32) demonstrated that BDNF changed synaptic connectivity in the SDH following peripheral nerve injury. Additionally, BDNF expression is regulated by TNF- $\alpha$ (32). Therefore, inhibition of inflammatory molecules and BDNF in spinal microglia by KCNMB3 siRNA may indirectly modulate synaptic connectivity culminating in attenuation of neuropathic pain.

Several molecules cause activation of microglia during neuropathic pain $(16,33)$. LPA (1-acyl-glycerol 3-phosphate) is one of the simplest phospholipids that serves as an extracellular signaling molecule. Following nerve injury, LPA is produced by activated neurons and facilitates its production in spinal microglia (33). LPA stimulation causes increased expression levels of BDNF in microglia (34). We previously identified that LPA induced robust activation of BK currents in spinal microglia, and BK channel inhibitor significantly attenuated allodynia-like behaviors, which was caused by intrathecal injection of LPA (3). A recent clinical study demonstrated that LPA concentration in the cerebrospinal fluid was correlated with pain symptoms in patients (35). Therefore, inhibition of BK channel function in microglia is potentially mediated through the LPA pathway in the spinal cord. In the present study, inhibition of microglia-specific BK channels did not completely abrogate neuropathic pain. This may be due to the involvement of factors other than LPA in activation of microglia and the generation of neuropathic pain $(17,33)$.

The effectiveness of ultra-low doses of paxilline, a BK channel-specific inhibitor, for epilepsy has been described (36). We also observed the effectiveness of paxilline in a model of morphine-induced hyperalgesia (8). Paxilline may cause side effects, although it effectively attenuated pathology. Paxilline selectively binds to the BK channel pore-gate domain and blocks channel activity. Previous evidence suggested that mice lacking KCNMA1, which encodes the pore-forming subunit of the BK channel, exhibit moderate ataxia (37); therefore, selective inhibition of BK channels in microglia is required for therapy. Given this evidence, KCNMB3, which is specifically expressed in microglia and regulates pain-associated molecules, is a promising therapeutic target for neuropathic pain.

In conclusion, the results of the present study demonstrated that a microglia-specific subtype of BK channels contributed to the induction of pro-inflammatory molecules and led to the generation of neuropathic pain. The identification of the role of BK channels improved understanding of microglial function and neuropathic pain.

\section{Acknowledgements}

Not applicable.

\section{Funding}

The present study was supported by a Grant-in-Aid for Scientific Research from the Japan for the Promotion of Science (JSPS) (JP16K11477 to YHay).

\section{Availability of data and materials}

All the data generated and analyzed the present study are available from the corresponding author upon reasonable request.

\section{Authors' contributions}

YHay designed and supervised the study. KI, YHar, JZ and YHay performed the experiments. KI, YHar, JZ, YM and YHay analyzed the data. YHay wrote the manuscript. All authors have read and approved the final manuscript.

\section{Ethics approval and consent to participate}

All animal experiments were performed using approved protocol (no., A27-187-0) and in accordance with the guidelines of Kyushu University. Mice were housed in accordance with the Institutional Animal Care and Use Committee regulations at Kyushu University.

\section{Patient consent for publication}

Not applicable.

\section{Competing interests}

The authors declare that they have no competing interests.

\section{References}

1. Tsuda M, Shigemoto-Mogami Y, Koizumi S, Mizokoshi A, Kohsaka S, Salter MW and Inoue K: P2X4 receptors induced in spinal microglia gate tactile allodynia after nerve injury. Nature 424: 778-783, 2003.

2. Coull JA, Beggs S, Boudreau D, Boivin D, Tsuda M, Inoue K, Gravel C, Salter MW and De Koninck Y: BDNF from microglia causes the shift in neuronal anion gradient underlying neuropathic pain. Nature 483: 1017-1021, 2005.

3. Hayashi Y, Kawaji K, Sun L, Zhang X, Koyano K, Yokoyama T, Kohsaka S, Inoue K and Nakanishi $\mathrm{H}$ : Microglial Ca(2+)-activated $\mathrm{K}(+)$ channels are possible molecular targets for the analgesic effects of S-ketamine on neuropathic pain. J Neurosci 31: 17370-17382, 2011.

4. Kawasaki Y, Zhang L, Cheng JK and Ji RR: Cytokine mechanisms of central sensitization: Distinct and overlapping role of interleukin-1beta, interleukin-6, and tumor necrosis factor-alpha in regulating synaptic and neuronal activity in the superficial spinal cord. J Neurosci 28: 5189-5194, 2008.

5. Masuda T, Tsuda M, Yoshinaga R, Tozaki-Saitoh H, Ozato K, Tamura T and Inoue K: IRF8 is a critical transcription factor for transforming microglia into a reactive phenotype. Cell Rep 1: 334-340, 2012.

6. Salkoff L, Butler A, Ferreira G, Santi C and Wei A: Highconductance potassium channels of the SLO family. Nat Rev Neurosci 7: 921-931, 2006.

7. Ferrini F, Trang T, Mattioli TA, Laffray S, Del'Guidice T, Lorenzo LE, Castonguay A, Doyon N, Zhang W, Godin AG, et al: Morphine hyperalgesia gated through microglia-mediated disruption of neuronal $\mathrm{Cl}(-)$ homeostasis. Nat Neurosci 16: 183-192, 2013.

8. Hayashi Y, Morinaga S, Zhang J, Satoh Y, Meredith AL, Nakata T, Wu Z, Kohsaka S, Inoue K and Nakanishi H: BK channels in microglia are required for morphine-induced hyperalgesia. Nat Commun 7: 11697, 2016.

9. Contreras GF, Neely A, Alvarez O, Gonzalez C and Latorre R: Modulation of BK channel voltage gating by different auxiliary $\beta$ subunits. Proc Natl Acad Sci USA 109: 18991-18996, 2012.

10. Brenner R, Chen QH, Vilaythong A, Toney GM, Noebels JL, and Aldrich RW: BK channel beta4 subunit reduces dentate gyrus excitability and protects against temporal lobe seizures. Nat Neurosci 8: 1752-1759, 2005. 
11. Gargiulo S, Greco A, Gramanzini M, Esposito S, Affuso A Brunetti A and Vesce G: Mice anesthesia, analgesia, and care, Part I: Anesthetic considerations in preclinical research. ILAR J 53: E55-E69, 2012.

12. Chaplan SR, Bach FW, Pogrel JW, Chung JM and Yaksh TL: Quantitative assessment of tactile allodynia in the rat paw. J Neurosci Methods 53: 55-63, 1994.

13. Kohno K, Kitano J, Kohro Y, Tozaki-Saitoh H, Inoue K and Tsuda M: Temporal kinetics of microgliosis in the spinal dorsal horn after peripheral nerve injury in rodents. Biol Pharm Bull 41: 1096-1102, 2018

14. Livak KJ and Schmittgen TD: Analysis of relative gene expression data using real-time quantitative PCR and the 2(-Delta Delta C(T)) method. Methods 25: 402-408, 2001.

15. Taylor AM, Mehrabani S, Liu S, Taylor AJ and Cahill CM: Topography of microglial activation in sensory- and affect-related brain regions in chronic pain. J Neurosci Res 95: 1330-335, 2017.

16. Griffin RS, Costigan M, Brenner GJ, Ma CH, Scholz J, Moss A, Allchorne AJ, Stahl GL and Woolf CJ: Complement induction in spinal cord microglia results in anaphylatoxin C5a-mediated pain hypersensitivity J Neurosci 27: 8699-8708, 2007.

17. Inoue $\mathrm{K}$ and Tsuda $\mathrm{M}$ : Microglia in neuropathic pain: Cellular and molecular mechanisms and therapeutic potential. Nat Rev Neurosci 19: 138-152, 2018.

18. Schilling $\mathrm{T}$ and Eder C: Microglial $\mathrm{K}(+)$ channel expression in young adult and aged mice. Glia 63: 664-672, 2015

19. Bordey A and Spencer DD: Chemokine modulation of high-conductance $\mathrm{Ca}(2+)$-sensitive $\mathrm{K}(+)$ currents in microglia from human hippocampi. Eur J Neurosci 18: 2893-2898, 2003.

20. Schilling $T$ and Eder $C$ : Ion channel expression in resting and activated microglia of hippocampal slices from juvenile mice. Brain Res 1186: 21-28, 2007.

21. Parzych K, Zetterqvist AV, Wright WR, Kirkby NS, Mitchell JA and Paul-Clark MJ: Differential role of pannexin-1/ATP/P2X7 axis in IL-1 $\beta$ release by human monocytes. FASEB J 31: 2439-2445, 2017.

22. Scheel O, Papavlassopoulos M, Blunck R, Gebert A, Hartung T, Zähringer U, Seydel U and Schromm AB: Cell activation by ligands of the toll-like receptor and interleukin-1 receptor family depends on the function of the large-conductance potassium channel MaxiK in human macrophages. Infect Immun 74: 4354-4356, 2006.

23. Boucsein C, Zacharias R, Färber K, Pavlovic S, Hanisch UK and Kettenmann H: Purinergic receptors on microglial cells: Functional expression in acute brain slices and modulation of microglial activation in vitro. Eur J Neurosci 17: 2267-2276, 2003.

24. Paolicelli RC, Bolasco G, Pagani F, Maggi L, Scianni M, Panzanelli P, Giustetto M, Ferreira TA, Guiducci E, Dumas L, et al: Synaptic pruning by microglia is necessary for normal brain development. Science 333: 1456-1458, 2011.

25. Ueno M, Fujita Y, Tanaka T, Nakamura Y, Kikuta J, Ishii M and Yamashita T: Layer V cortical neurons require microglial support for survival during postnatal development. Nat Neurosci 16 : $543-551,2013$
26. Zeng X, Xia XM and Lingle CJ: Species-specific differences among KCNMB3 BK beta3 auxiliary subunits: Some beta3 $\mathrm{N}$-terminal variants may be primate-specific subunits. J Gen Physiol 132: 115-129, 2008.

27. Lee US and Cui J: \{beta\} subunit-specific modulations of BK channel function by a mutation associated with epilepsy and dyskinesia. J Physiol 587: 1481-1498, 2009.

28. Masuda T, Ozono Y, Mikuriya S, Kohro Y, Tozaki-Saitoh H, Iwatsuki K, Uneyama H, Ichikawa R, Salter MW, Tsuda M and Inoue K: Dorsal horn neurons release extracellular ATP in a VNUT-dependent manner that underlies neuropathic pain. Nat Commun 7: 12529, 2016.

29. Mizoguchi Y, Kato TA, Seki Y, Ohgidani M, Sagata N, Horikawa H, Yamauchi Y, Sato-Kasai M, Hayakawa K, Inoue R, et al: Brain-derived neurotrophic factor (BDNF) induces sustained intracellular $\mathrm{Ca} 2+$ elevation through the up-regulation of surface transient receptor potential 3 (TRPC3) channels in rodent microglia. J Biol Chem 289: 18549-18555, 2014

30. Tay TL, Mai D, Dautzenberg J, Fernández-Klett F, Lin G, Sagar, Datta M, Drougard A, Stempfl T, Ardura-Fabregat A, et al: A new fate mapping system reveals context-dependent random or clonal expansion of microglia. Nat Neurosci 20: 793-803, 2017.

31. Parkhurst CN, Yang G, Ninan I, Savas JN, Yates JR III, Lafaille JJ, Hempstead BL, Littman DR and Gan WB: Microglia promote learning-dependent synapse formation through brain-derived neurotrophic factor. Cell 155: 1596-1609, 2013.

32. Liu Y, Zhou LJ, Wang J, Li D, Ren WJ, Peng J, Wei X, Xu T, Xin WJ, Pang RP, et al: TNF- $\alpha$ differentially regulates synaptic plasticity in the hippocampus and spinal cord by microglia-dependent mechanisms after peripheral nerve injury. J Neurosci 37: 871-881, 2017

33. Ueda H, Matsunaga H, Olaposi OI and Nagai J: Lysophosphatidic acid: Chemical signature of neuropathic pain. Biochim Biophys Acta 1831: 61-73, 2013.

34. Fujita R, Ma Y and Ueda H: Lysophosphatidic acid-induced membrane ruffling and brain-derived neurotrophic factor gene expression are mediated by ATP release in primary microglia. J Neurochem 107: 152-160, 2008.

35. Kuwajima K, Sumitani M, Kurano M, Kano K, Nishikawa M, Uranbileg B, Tsuchida R, Ogata T, Aoki J, Yatomi Y and Yamada Y: Lysophosphatidic acid is associated with neuropathic pain intensity in humans: An exploratory study. PLoS One 13: e0207310, 2018.

36. Liu J, Ye J,Zou X, Xu Z, Feng Y, Zou X, Chen Z, Li Y and Cang Y: CRL4A(CRBN) E3 ubiquitin ligase restricts BK channel activity and prevents epileptogenesis. Nat Commun 5: 3924, 2014.

37. Meredith AL, Thorneloe KS, Werner ME, Nelson MT and Aldrich RW: Overactive bladder and incontinence in the absence of the BK large conductance $\mathrm{Ca} 2+-$ activated $\mathrm{K}+$ channel. J Biol Chem 279: 36746-36752, 2004 